\title{
Adverse reaction to ceftriaxone in a 28-day-old infant undergoing urgent craniotomy due to epidural hematoma: review of neonatal biliary pseudolithiasis
}

\author{
This article was published in the following Dove Press journal: \\ Therapeutics and Clinical Risk Management \\ 2 July 2015 \\ Number of times this article has been viewed
}

\author{
Alicja Bartkowska- \\ Śniatkowska' \\ Katarzyna Jończyk- \\ Potoczna ${ }^{2}$ \\ Marzena Zielińska ${ }^{3}$ \\ Jowita Rosada-Kurasińska' \\ 'Department of Pediatric \\ Anesthesiology and Intensive Therapy, \\ Poznan University of Medical Sciences, \\ Poznan, Poland; '2Department of \\ Pediatric Radiology, Poznan University \\ of Medical Sciences, Poznan, Poland; \\ ${ }^{3}$ Department of Anesthesiology and \\ Intensive Care, Pediatric Intensive \\ Care Unit, Wroclaw Medical \\ University, Wroclaw, Poland
}

\begin{abstract}
The debate as to whether to administer ceftriaxone to neonates is likely to continue. Ceftriaxone has numerous advantages for critically ill pediatric patients. However, it is also known to contribute substantially to the development of biliary pseudolithiasis. Although pediatric patients rarely develop gallbladder disorders, this complication may lead to adverse events in high-risk patients with predisposing factors, particularly in neonates and infants treated with ceftriaxone. In this paper we present an interesting case report of a 28 -day-old neonate with spontaneous severe epidural hematoma who developed biliary pseudolithiasis related to the use of ceftriaxone. We also discuss the efficacy of ceftriaxone in neonates and infants. Neonatologists and pediatric intensivists should be aware of the higher risk of co-existence of hyperbilirubinemia and gallbladder disorders while using ceftriaxone in pediatric settings.
\end{abstract}

Keywords: ceftriaxone, pharmacodynamics, neonate, pseudolithiasis, efficacy

\section{Introduction}

Recently, the pharmacokinetic and pharmacodynamic properties of a number of drugs have been studied in the pediatric population, including critically ill patients in neonatal and pediatric intensive care units. However, although the number of clinical studies has been increasing, there are still limited trial data on neonates and infants. ${ }^{1-5}$ For this reason, many questions regarding the individual properties of these drugs and their safety in the youngest, critically ill patients remain unanswered.

Antibiotics are a complex group of drugs and are commonly administered to children being managed in critical care. $\beta$-Lactam antibiotics, including cephalosporins, have an important role in the treatment of a variety of severe bacterial infections in infants and children. However, widespread use of these drugs may come with not only a higher risk of resistance but also a higher risk of adverse effects. ${ }^{6-11}$ Ceftriaxone, a third-generation cephalosporin, is commonly used for the treatment of severe infections in critically ill pediatric patients due to its advantages ie, a broad spectrum of activity, substantial resistance to $\beta$-lactamases, good penetration into tissues, predictable and adequate plasma concentrations for therapeutic effect, and a prolonged halflife. Ceftriaxone is highly bound to plasma proteins (97\%), but in infants can displace bilirubin from its protein binding sites and exacerbate physiological jaundice. The kidneys (60\%) and liver (40\%) excrete this drug, but in patients with impaired renal function, biliary excretion may be increased. The rate of complications related to use of this drug may be higher in newborns, particularly those with low or very low birth
Correspondence: Alicja BartkowskaŚniatkowska

Department of Pediatric Anesthesiology and Intensive Therapy, Poznan University of Medical Sciences, Szpitalna Street 27/33, Poznan 60-572, Poland

Tel +48 6I 849 I478

Fax +48 6I 849 I486

Email asniatko@ump.edu.pl 
weight, and may require intensive care. ${ }^{12,13}$ Age older than 24 months, Gram-negative sepsis, reduced visceral flow due to hypovolemic shock, total parenteral nutrition followed by reduced flow of bile prior surgery, hypercalcemia, and longterm treatment with high doses $(>100 \mathrm{mg} / \mathrm{kg} /$ day $)$ are the most common predisposing factors. The most common side effects associated with administration of ceftriaxone include allergic reactions (rash, eosinophilia, fever, anaphylactic shock), gastrointestinal disturbances, a transient increase in transaminases, hematological abnormalities (granulocytopenia, thrombocytopenia, hemolytic anemia) and gallbladder resolution deficiency. ${ }^{14-16}$ Gökçe et al suggested that the distribution of risk factors depends on both medical and non-medical changes, including geographic location, facilities available, and the reference status of the center. ${ }^{15}$ Cholelithiasis, increased biliary sludge, and pseudolithiasis rarely occur in childhood, but there is a bimodal distribution characterized by two peaks, the first being in infancy and the second in early adolescence. ${ }^{15,17}$ In current practice, due to increased use of radiological imaging such as ultrasonography in neonatal and pediatric intensive care units this condition can be diagnosed more often. ${ }^{18-20}$ These diagnostic methods have contributed to an increased detection rate of this phenomenon, even in the youngest patients in pediatric intensive care unit, who may be asymptomatic.

This report describes specific considerations concerning the efficacy of ceftriaxone, a third-generation cephalosporin, in neonatal and pediatric practice, and follows the course of treatment of a 28-day-old neonate with life-threatening epidural bleeding followed by biliary pseudolithiasis due to administration of ceftriaxone as perioperative antibiotic prophylaxis.

\section{Case report}

A 28-day-old infant boy was referred from the district to the University Children's Hospital to diagnose and treat severe spontaneous epidural bleeding, which was the original diagnosis. He was the fourth child of young parents, born from the fourth pregnancy, which ended at week 42. During the first 4 weeks of life, he did not show any symptoms of a congenital defect or any significant comorbidity.

On admission to the emergency department of a regional hospital, he was anxious and crying which was followed by a deep coma with a Glasgow Coma Scale score decreasing from 7 to 3 points. Anisocoria, bradycardia of 80-100 beats per minute, and bradypnea of 15 breaths per minute were also observed. Laboratory investigations revealed severe anemia and significant coagulopathy. No traumatic event or external action involving another person was reported in the anamnesis. Computerized tomography of the head revealed an intracranial hemorrhage and mass effect due to severe epidural hematoma of $0.11 \mathrm{~cm}$ with disseminated ischemic foci. The patient underwent urgent extensive frontoparietal craniotomy performed as a life-saving procedure by surgeons in the regional hospital. Broad-spectrum antibiotic prophylaxis was considered and ceftriaxone seemed to be the best choice. Ceftriaxone was administered intravenously at a dose of $100 \mathrm{mg} / \mathrm{kg}$ once a day. When the patient's clinical condition was deemed to be compatible with stabilization of the respiratory and circulatory systems, he was transferred to the University Hospital for Children to continue advanced intensive neurosurgical treatment.

On admission to the pediatric intensive care unit, the severity of the neonate's illness was assessed at 20 points according to PRISM (Pediatric Risk of Mortality), with a predicted risk of death of $34.4 \%$. He was put into a barbiturate-induced coma as a neuroprotective strategy with supplementary sedation and analgesia due to the need for mechanical ventilation as a result of postoperative respiratory insufficiency. The patient was ventilated to normocapnia with pressure-controlled ventilation and parameters corresponding to his age and respiratory condition (Maquet ventilator, DrägerWerke AG, Lübeck, Germany). Empirical antibiotic therapy with ceftriaxone was continued, used previously as prophylaxis ( $100 \mathrm{mg} / \mathrm{kg}$ every 24 hours $)$, because of its excellent penetration into the cerebrospinal fluid (CSF). The choice of ceftriaxone from the third-generation cephalosporins was consistent with the recommendations at our institution.

Laboratory investigations revealed deep, normocytic (mean corpuscular volume 82.9-84.6 fl) and normochromic (mean corpuscular hemoglobin concentration 34.4-36.9 g/dL) anemia (hemoglobin $7.8 \mathrm{~g} / \mathrm{dL}$, hematocrit $23 \%$ ) as an effect of severe brain hemorrhage and blood loss. Additional results revealed a slightly elevated level of total bilirubin $(3.7 \mathrm{mg} / \mathrm{dL})$, a lower total protein $(4.4 \mathrm{~g} / \mathrm{dL})$, and normal aspartate transaminase (36 IU/L) and alanine transaminase (11 IU/L) (Table 1). The coagulation screen showed normal antithrombin activity (70\%) with an increased international normalized ratio (3.57) and a decreased prothrombin time (60\%). Protein C and protein S levels were 44.7 U/dL and $98.8 \mathrm{U} / \mathrm{dL}$, respectively. The patient required transfusions of red blood cells, fresh frozen plasma, and vitamin $\mathrm{K}$ supplementation. At the same time, a number of metabolic investigations were carried out in search of the primary cause of the unexplained intracranial bleeding. However, these excluded any congenital metabolic disorder. 
Table I Mean total bilirubin, direct bilirubin, albumin, ALT, and AST levels

\begin{tabular}{lllll}
\hline & Day I & Day 2 & Day 7 & Day I4 \\
\hline Bilirubin total (mg/dL) & 3.79 & 3.37 & 5.89 & 8.38 \\
Bilirubin direct (mg/dL) & 1.72 & 2.14 & 3.31 & 4.52 \\
Albumin (g/dL) & 2.48 & NA & 2.42 & NA \\
ALT (IU/L) & 11 & 12 & 21 & 46 \\
AST (IU/L) & 36 & 40 & 78 & 104 \\
\hline
\end{tabular}

Abbreviations: ALT, alanine transaminase; AST, aspartate transaminase; NA, not available.

On the second day of hospital stay, the patient underwent computerized tomography of the head and ultrasound imaging of his abdomen as a routine pediatric intensive care procedure. Post-craniotomy axial computerized tomography scans revealed extensive hypodensity involving the gray as well as the white matter of the left hemisphere with bleeding foci. Midline shift and uncal herniation were also seen (Figure 1). Also the following sonographic findings were observed in the abdomen: dilation of the biliary tree, thickening of the dilated cystic duct wall, common hepatic duct, and common bile duct (Figure 2A) and mass-like sludge in the gallbladder (Figure 2B).

In view of the ceftriaxone therapy, the picture of biliary sludge suggested ceftriaxone-associated pseudolithiasis. Ceftriaxone was discontinued on the following day, after a total therapy that lasted 3 days. At this time, the ursodeoxycholic acid was administered to the child at a dose of $15 \mathrm{mg} / \mathrm{kg}$ twice a day and fat-soluble vitamins as supplementary therapy.

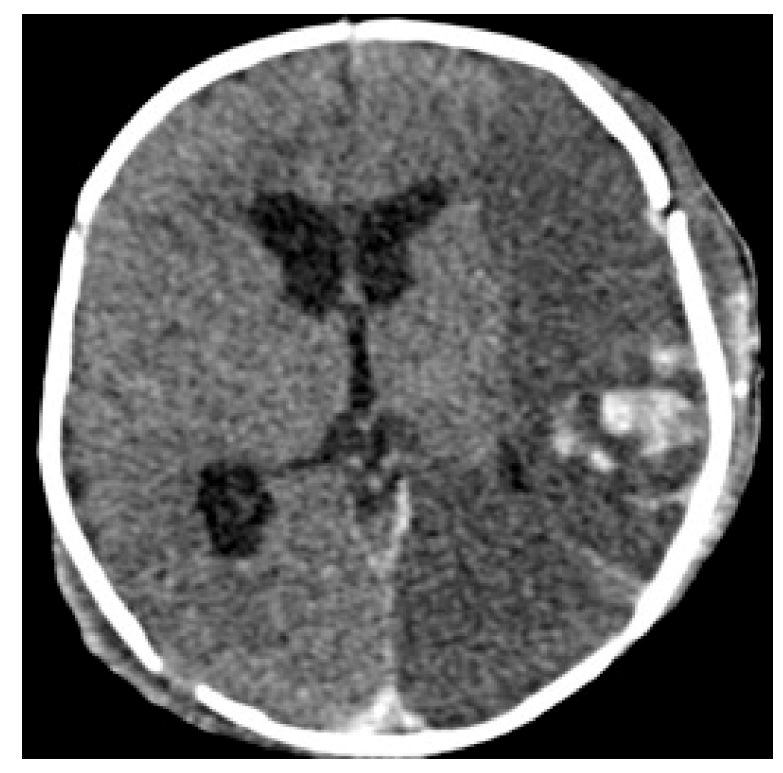

Figure I Post craniotomy axial CT shows extensive hypodensity involving the gray and white matter of the left hemisphere with bleeding foci. Midline shift and uncal herniation is also seen.
After 2 weeks of treatment, ultrasonography revealed partial and gradual improvement and no surgical intervention was required (Figure 3). Following another week, ultrasound examination showed complete normalization of image (Figure 4). There was a gradual improvement of the radiological image over the course of 3 weeks and the bilirubin remained elevated for the first 14 days, reaching a maximum level of $8.38 \mathrm{mg} / \mathrm{dL}$. Nevertheless, due to the prolonged hyperbilirubinemia, phototherapy was also introduced for the next 3 days, but no spectacular improvement was achieved. Despite this drug-related complication, the infant's neurological status gradually improved, and he was able to be weaned and disconnected from the ventilator. He was successfully extubated on day 12 of his stay in pediatric intensive care. The first neurological examination was made when the child was conscious (Glasgow Coma Scale score of 12 points) and revealed signs of right-handed paresis. The patient was transferred to the surgical department to continue treatment and rehabilitation.

\section{Discussion}

Similarly to other third-generation cephalosporins, ceftriaxone has many attractive properties as an antibiotic ie, a broad spectrum of action, a long half-life, once-a-day dosing, and good tissue penetration in the central nervous system. This drug has proved to be clinically effective, relatively safe and easy to use so is the most common antibiotic used as an initial empiric therapy for critically ill patients in intensive care. ${ }^{21}$ It may be difficult to diagnose pathogens especially in neonatal intensive care unit and due to the absence of such data, local epidemiology and susceptibility patterns may contribute to the selection of empiric therapy. The latest evidence-based recommendation is that ceftriaxone should be used as an initial therapy because of its broad spectrum of action and favorable benefit-risk ratio. ${ }^{22}$ For these reasons, our patient was a candidate for empiric ceftriaxone therapy after craniotomy for a very severe brain injury.

The World Health Organization (WHO) and US Food Drug Administration (FDA) suggest a combination of ceftriaxone and gentamicin for the treatment of neonates aged 0-28 days who suffer from a severe illness. ${ }^{23,24}$ The advantages of ceftriaxone in neonates with severe infection include its high effectiveness, good tolerance and relative safety. The doses recommended for neonates younger than 72 hours are lower $(50 \mathrm{mg} / \mathrm{kg})$ than those administered to infants older than 28 days $(100 \mathrm{mg} / \mathrm{kg}) .{ }^{25}$ These higher doses were used in our patient due to the excellent blood-brain barrier penetration. 


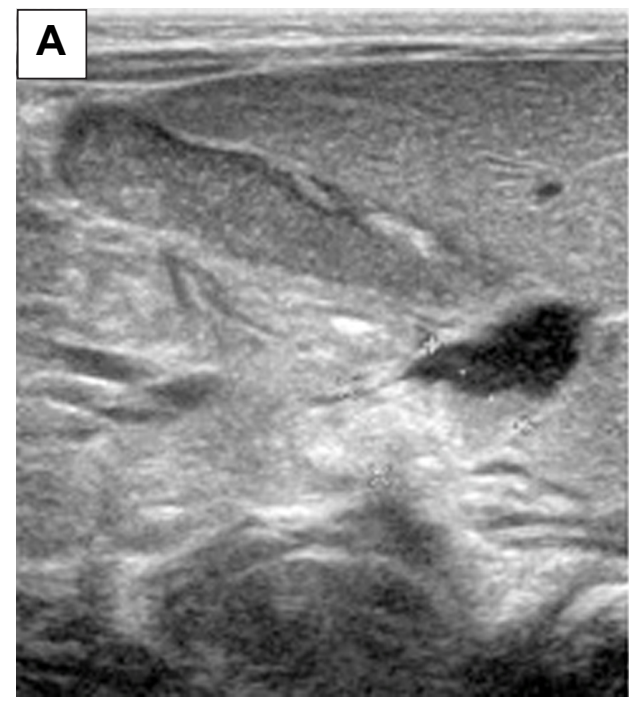

Figure 2 Mass-like sludge in common bile duct (A) and the gallbladder (B).

Both the WHO and FDA do not recommend simultaneous administration of ceftriaxone and solutions containing calcium to neonates, especially via intravenous infusion and using the same catheter line because of the risk of precipitation of ceftriaxone-calcium salts in the lungs and kidneys. Recent in vitro studies of neonatal and adult plasma did not reveal a direct correlation between ceftriaxone and infusions of calcium-containing solutions. This allowed the FDA in 2009 to modify its earlier restriction for ceftriaxone administration in both adult and pediatric patients $(>28$ days). The most recent recommendation is based on the Safety Information and Adverse Event Reporting Program. ${ }^{26}$

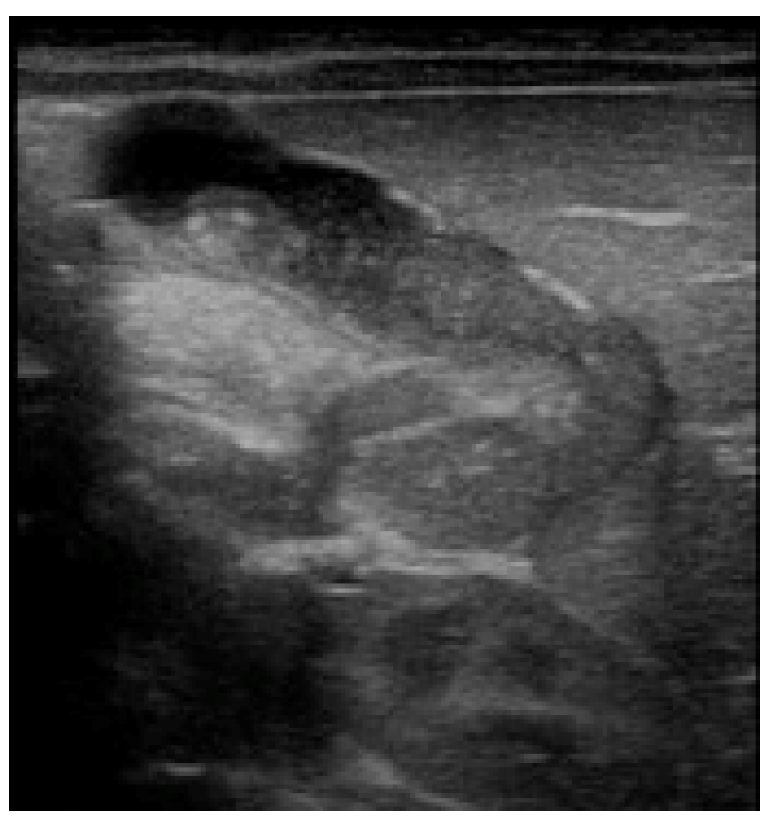

Figure 3 The gallbladder after 2 weeks of treatment - partial and gradual improvement.

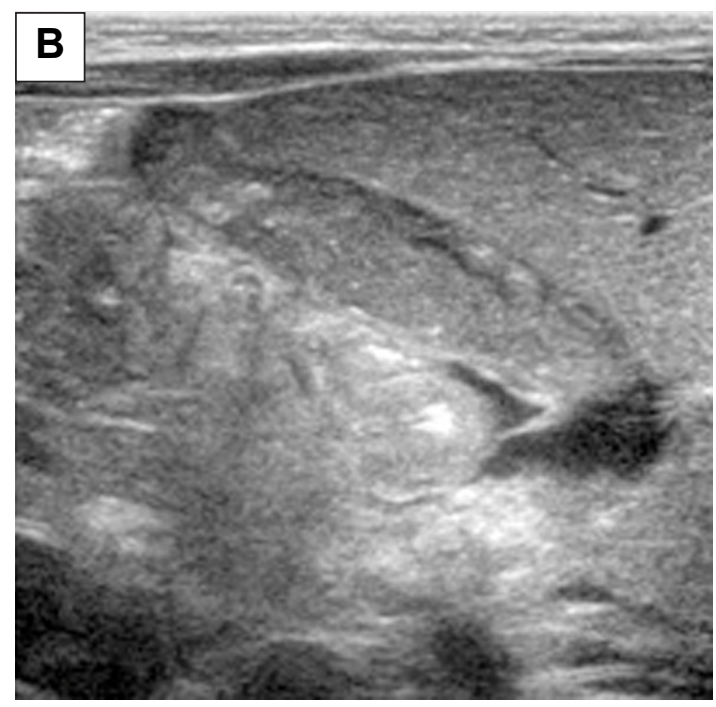

The main change did ease the restriction of the sequential (48 hours) and separate administration of ceftriaxone and calcium-containing fluids to avoid calcium precipitation and to minimize serious organ toxicity caused by ceftriaxonecalcium precipitates. ${ }^{6,27}$ Concomitant use of ceftriaxone and intravenous calcium-containing products is still contraindicated in neonates ( $\leq 28$ days of age). In this study, the patient's serum calcium was normal and calcium-containing fluids were administered separately.

Hyperbilirubinemia is an important contraindication to administration of ceftriaxone in neonates, especially preterm newborns, because of the displacement of bilirubin from albumin-binding sites and an increase in serum concentrations of free bilirubin. However, risk factors for biliary

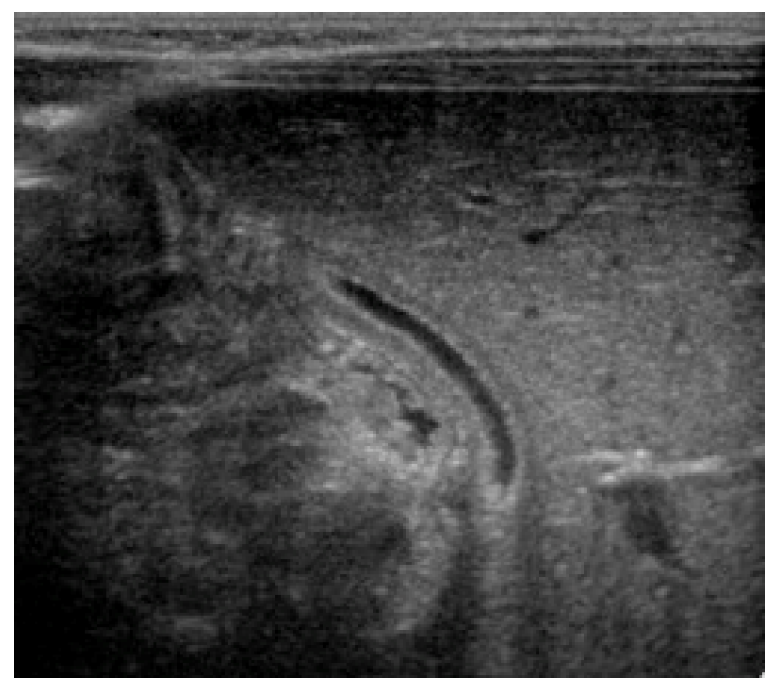

Figure 4 The gallbladder after 3 weeks of treatment - complete normalization of image. 
pseudolithiasis include age older than 24 months and higher doses of ceftriaxone ( $\geq 2 \mathrm{~g} /$ day) used as a long-term treatment. Neonates and infants in particular are at higher risk of a poor outcome because of bilirubin encephalopathy. ${ }^{28-30}$ Therefore, intensivists should be aware of hyperbilirubinemia, pseudolithiasis and biliary sludge as an adverse reaction to ceftriaxone therapy in neonatal and pediatric intensive care unit patients. Indeed, our patient demonstrated slightly elevated bilirubin levels on the second day of treatment with ceftriaxone, but this therapy was started earlier in the surgical department as antibiotic prophylaxis and was only discontinued following an abnormal ultrasound examination. Gulian et al investigated physiological jaundice in newborns and examined three forms of bilirubin, ie, bound to albumin, unbound, and linked to cellular membranes (to erythrocytes in particular). The authors found a significantly increased number of bilirubin-erythrocyte complexes after exposure to ceftriaxone. Furthermore, the study has shown that binding of bilirubin by erythrocytes could be used as a determinant of bilirubin displacement by drugs. ${ }^{28}$

Ceftriaxone-induced pseudolithiasis and biliary sludge were first described by Schaad et al. ${ }^{30,31}$ Since that time, numerous case reports and prospective studies have described the relationship between ceftriaxone, pseudolithiasis, and biliary sludge in both adults and children. ${ }^{16,32-34}$ To our knowledge, there are still limited data concerning this phenomenon in neonates and infants, requiring specific factors that are typical for this extreme age group of patients. ${ }^{12}$ Firstly, biliary cholelithiasis and nephrolithiasis are asymptomatic and diagnosed by means of sonographic examination rather than by laboratory tests or clinical signs. ${ }^{35,36}$ Secondly, it may be impossible to diagnose an adverse event in the neonatal and pediatric setting using the Naranjo adverse drug reaction probability scale despite its usefulness in adults. Some questions included in this paper, ie, those assessing retrospective events related to drugs used previously as well as assessing previous reports of adverse patient-drug reactions remained unanswered. Finally, re-administration of the same potentially toxic drug in this group of patients requires further research as it is not fully explained. ${ }^{37}$

Unlike biliary pseudolithiasis, nephrolithiasis induced by kidney calcifications occurs much more rarely in children treated with ceftriaxone. The mechanism of formation of ceftriaxone precipitates in the kidneys can be explained with an elevated level of urinary uric acid. Moreover, being an organic anion, ceftriaxone behaves like a calciumsensitive anion, which is implicated in the development of nephrolithiasis. ${ }^{38}$
The pathogenesis of biliary and urinary precipitates has not been well investigated yet. The fact that only a limited number of patients treated with ceftriaxone develop pseudolithiasis indicates that individual predisposition does exist, particularly in the genetic profile. Fratzayas et al studied three pediatric patients aged 5.5, 18, and 48 months, who were receiving ceftriaxone at doses of $100 \mathrm{mg} / \mathrm{kg} /$ day and developed pseudolithiasis in the gallbladder. ${ }^{39}$ All of these patients were carriers of the A(TA)7TAA polymorphism of the UGT1A1 gene encoding uridine diphosphate (UDP)-glucuronosyltransferase, the enzyme involved in glucuronidation of bilirubin.

Although ceftriaxone can interact with calcium and displace bilirubin from albumin-binding sites, increasing the risk of adverse events, one may still consider use of the drug in the neonatal period due to the considerable CSF concentrations reached. ${ }^{21}$ Thus, it is possible to extend the indications to the youngest patients with primary brain injury, particularly after neurosurgical procedures. The pharmacokinetic properties of ceftriaxone are of interest due to the high drug concentrations reaching the CSF after intravenous administration. Steele et al found ceftriaxone concentrations in the CSF of infants to be $5.4 \mu \mathrm{g} / \mathrm{mL}$ and $6.4 \mu \mathrm{g} / \mathrm{mL}$ after doses of $50 \mathrm{mg} / \mathrm{kg}$ and $70 \mathrm{mg} / \mathrm{kg}$, respectively, with CSF to peak serum concentrations of $2.2 \%-2.3 \% .^{40} \mathrm{McC}$ racken et al assessed ceftriaxone in newborns weighing less than 1,500 $\mathrm{g}$ and observed significant differences ie, the mean plasma half-life was longer and the volume of distribution was larger. No other differences were found in older neonates and infants. ${ }^{13}$ However, the clearance of ceftriaxone is highly dependent on developmental age and the clearance of cephalosporin increases as postnatal maturation proceeds. ${ }^{21}$

The major limitation of this study is the lack of monitoring of plasma ceftriaxone concentration. However, biliary pseudolithiasis appeared as an unexpected adverse event very early on and ceftriaxone was discontinued after the third dose (48 hours after the start of therapy) of the drug. Cephalosporins like other $\beta$-lactams, macrolides and quinolones have a wide therapeutic index $x^{41,42}$ and it is neither recommended nor possible to monitor plasma concentrations of cephalosporins in standard daily practice.

\section{Conclusion}

Ceftriaxone can cause transient reversible biliary pseudolithiasis infrequently but rarely causes nephrolithiasis or ceftriaxone-calcium precipitates in the lungs and kidneys. The incidence of this phenomenon in the pediatric population has been reported to be in the wide range from 3\% to $50 \%$, particularly in older children receiving higher doses 
of ceftriaxone ( $>2 \mathrm{~g} / \mathrm{kg} /$ day) as long-term therapy (5-6 days) with spontaneous disappearance of symptoms within 1-2 weeks after cessation of the drug administration. ${ }^{30,43,44}$ Neonates and infants are at higher risk of hyperbilirubinemia and are more vulnerable to the side effects of ceftriaxone which include pseudolithiasis and biliary sludge that can occur even in the first 2-3 days of therapy. Further research is necessary to ensure that the dosing regimens of ceftriaxone used in neonates with brain injury are entirely evidencebased. ${ }^{21}$ Neonatologists and pediatric intensivists need to be aware of the higher risk of hyperbilirubinemia and gallbladder disorders associated with ceftriaxone therapy. These conditions require adequate monitoring and early detection. The protocol of repeat ultrasound monitoring seems to be the gold standard for neonates and infants undergoing ceftriaxone therapy and having a higher risk of hyperbilirubinemia and biliary pseudolithiasis. ${ }^{44,45}$

\section{Disclosure}

The authors report no conflicts of interest in this work.

\section{References}

1. Blumer JL. Clinical pharmacology of midazolam in infants and children. Clin Pharmacokinet. 1998;35(2):37-47.

2. Peeters MY, Prins SA, Knibbe CA, et al. Pharmacokinetics and pharmacodynamics of midazolam and metabolites in nonventilated infants after craniofacial surgery. Anesthesiology. 2006;105(6):1135-1146.

3. de Wildt SN, de Hoog M, Vinks AA, van der Giesen E, van den Anker JN. Population pharmacokinetics and metabolism of midazolam in pediatric intensive care patients. Crit Care Med. 2003;31(7):1952-1958.

4. Davis PJ, Cook DR, Stiller RL, Davin-Robinson KA. Pharmacodynamics and pharmacokinetics of high-dose sufentanil in infants and children undergoing cardiac surgery. Anesth Analg. 1987;66(3):203-208.

5. Woloszczuk-Gebicka B, Grabowski T, Borucka B, Karas-Trzeciak M. Pharmacokinetics of sufentanil administered with $0.2 \%$ ropivacaine as a continuous epidural infusion for postoperative pain relief in infants. Paediatr Anaesth. 2014;24(9):962-967.

6. Bradley JS, Wassel RT, Lee L, Nambiar S. Intravenous ceftraxione and calcium in the neonate: assessing the risk for cardiopulmonary adverse events. Pediatrics. 2009;123(4):609-613.

7. Monte SV, Prescott WA, Johnson KK, Kuhman L, Paladino JA. Safety of ceftriaxone sodium at extremes of age. Expert Opin Drug Saf. 2008;7(5): $515-523$.

8. Yao Y, Zhou R, Wang Y. Fatal adverse effects of injected ceftriaxone sodium in China. Pharmacoepidemiol Drug Saf. 2012;21(11): $1197-1201$.

9. Atanasković-Marković M, Velicković TC, Gavrović-Jankulović M, Vucković O, Nestorović B. Immediate allergic reactions to cephalosporins and penicillins and their cross-reactivity in children. Pediatr Allergy Immunol. 2005;16(4):341-347.

10. Belliard CR, Sibille G. Anaphylactoid shock or precipitation of calciumceftriaxone in a premature newborn. A case report. Arch Pediatr. 2007; 14(2):199-200.

11. Romano A, Caubet JC. Antibiotic allergies in children and adults: from clinical symptoms to skin testing diagnosis. J Allergy Clin Immunol Pract. 2014;2(1):3-12.

12. Martin E, Fanconi S, Kälin P, et al. Ceftriaxone-bilirubin-albumin interactions in the neonate an in vivo study. Eur J Pediatr. 1993;152(6): $530-534$.
13. McCracken GH Jr, Siegel JD, Threlkeld N, Thomas M. Ceftriaxone pharmacokinetics in newborn infants. Antimicrob Agents Chemother. 1983; 23(2):341-343.

14. European Medicines Agency. Annex III. Summary of product characteristics, labelling and package leaflet. Available from: http://www. ema.europa.eu/docs/en_GB/document_library/Referrals_document/ Rocephin_30/WC500160113.pdf. Accessed May 15, 2015.

15. Gökçe S, Yildirim M, Erdoğan D. A retrospective review of children with gallstones: single-center experience from Central Anatolia. Turk J Gastroenterol. 2014;25(1):46-53.

16. Rivkin AM. Hepatocellular enzyme elevations in a patient receiving ceftriaxone. Am J Health Syst Pharm. 2005;62(19):2006-2010.

17. Alehossein M, Sotoudeh K, Nasoohi S, Salamati P, Akhtare-Khavari H. Ceftriaxone induced biliary pseudocholelithiasis in children. Report of 14 cases. Iran J Pediatr. 2008;18(1):31-37.

18. Soysal A, Erasov K, Akpinar I, Bakir M. Biliary precipitation during ceftriaxone therapy: frequency and risk factors. Turk J Pediatr. 2007; 49(4):404-407.

19. Biner B, Oner N, Celtik C, et al. Ceftriaxone-associated biliary pseudolithiasis in children. J Clin Ultrasound. 2006;34(5):217-222.

20. Kutuya N, Ozaki Y, Okazaki T. Symptomatic child with ceftriaxoneassociated biliary pseudocholithiasis. J Med Ultrason (2001). 2008;35: 125-128.

21. Pacifici GM. Pharmacokinetics of cephalosporins in the neonate: a review. Clinics (Sao Paulo). 2011;66(7):1267-1274.

22. Hamilton JL, John SP. Evaluation of fever in infants and young children. Am Fam Physician. 2013;87(4):254-260.

23. http://www.fda.gov. Available from: http://www.fda.gov/downloads/ Safety/MedWatch/safetyInformation/Safetyalertsforhumanmedicalproducts/ucm165639.pdf. Accessed May 15, 2015.

24. World Health Organization. Ceftriaxone - safety in neonates. Available from: http://www.who.int/selection_medicines/committees/ subcommittee/2/Ceftriaxone.pdf. Accessed May 15, 2015.

25. Van Reempts PJ, Van Overmeire B, Mahieu LM, Vancker KJ. Clinical experience with ceftriaxone treatment in the neonate. Chemotherapy. 1995;41(4):316-322.

26. Bradley JS, Bocchini JA. FDA eases restrictions on use of ceftriaxone in infants. AAP News. 2009;30:28. DOI: 10.1542/aapnews. 2009306-28

27. Steadman E, Raisch DW, Bennett CL, et al. Evaluation of a potential interaction between ceftriaxone and calcium. Antimicrob Agents Chemother. 2010;54(4):1534-1540.

28. Gulian JM, Dalmasso C, Pontier F, Gonard V. Displacement effect of ceftriaxone on bilirubin bound to human serum albumin. Chemotherapy. 1986;32(5):399-403.

29. Abu Teir MM, Ghitan J, Abu-Taha MI, Darwish SM, Abu-Hadid MM. Spectroscopic approach of the interaction study of ceftriaxone and human serum albumin. J Biophys Struct Biol. 2014;6(1):1-12.

30. Schaad UB, Tschäppeler H, Lentze NJ. Transient formation of precipitations in the gallbladder associated with ceftriaxone therapy. Pediatr Infect Dis. 1986;5(6):708-710.

31. Schaad UB, Wedgwood-Krucko J, Tschäppeler H. Reversible ceftriaxone-associated biliary pseudolithiasis in children. Lancet. 1988; 2(8625):1411-1413.

32. Alam MK, Baslul Karim ASM, Kabir MH, Huque SS, Samsuzzaman M. Ceftriaxone associated biliary sludge in children a study in Bangabandhu Sheikh Mujib Medical University. Bangladesh J Child Health.2013;37(3):142-145.

33. Bickford CL, Spencer AP. Biliary sludge and hyperbilirubinemia associated with ceftriaxone in an adult: case report and review of the literature. Pharmacotherapy. 2005;25(10):1389-1395.

34. Schaad UB, Stoeckel K. Single-dose pharmacokinetics of ceftriaxone in infants and young children. Antimicrob Agents Chemother. 1982;21(2): 248-253.

35. Prince JS, Senac MO Jr. Ceftriaxone-associated nephrolithiasis and biliary pseudolithiasis in a child. Pediatr Radiol. 2003;33(9): 648-651. 
36. Miloh T, Kotlus Rosenberg H, Kochin I, Kerkar N. Inspissated bile syndrome in a neonate treated with ceftriaxone. J Ultrasound Med. 2009;28: 541-544.

37. Naranjo CA, Busto U, Sellers EM, et al. A method for estimating the probability of adverse drug reactions. Clin Pharmacol Ther. 1981;30(2): 239-245.

38. Akl KF, Masri AT, Hjazeen MM. Acute urinary retention induced by ceftriaxone. Saudi J Kidney Dis Transpl. 2011;22(6):1226-1228.

39. Fratzayas A, Liapi O, Papadopoulou A, Nicolaidou P, Stamoulakatou A. Is ceftriaxone-induced biliary pseudocholithiasis influenced by UDPglucuronosyltransferase 1A1 gene polymorphisms? Case Rep Med. 2011;2011:730250.

40. Steele RW, Eyre LB, Bradsher RW, Weinfeld RE, Patel IH, Spicehandler J. Pharmacokinetics of ceftriaxone in pediatric patients with meningitis. Antimicrob Agents Chemother. 1983;23(2):191-194.
41. Hammett-Stabler CA, Johns T. Laboratory guidelines for monitoring of antimicrobial drugs. Clin Chem. 1998;44(5):1129-1140.

42. Begg EJ, Barclay ML, Kirkpatrick CJM. The therapeutic monitoring of antimicrobial agents. Br J Clin Pharmacol. 1999;47(1):23-30.

43. Papadopoulou F, Efremidis S, Karyda S, et al. Incidence of ceftriaxone-associated gallbladder pseudolithiasis. Acta Paediatr. 1999; $88: 1352-1355$.

44. Alemayehu H, Desai AA, Thomas P, Sharp SW, St Peter SD. Ceftriaxone-induced pseudolithiasis in children treated for perforated appendicitis. Pediatr Surg Int. 2014;30(3):323-32

45. Andruszkiewicz P, Sobczyk D. Ultrasound in critical care. Anaesthesiol Intensive Ther. 2013;45:177-181.

\section{Publish your work in this journal}

Therapeutics and Clinical Risk Management is an international, peerreviewed journal of clinical therapeutics and risk management, focusing on concise rapid reporting of clinical studies in all therapeutic areas, outcomes, safety, and programs for the effective, safe, and sustained use of medicines. This journal is indexed on PubMed Central, CAS,
EMBase, Scopus and the Elsevier Bibliographic databases. The manuscript management system is completely online and includes a very quick and fair peer-review system, which is all easy to use. Visit http://www.dovepress.com/testimonials.php to read real quotes from published authors.

Submit your manuscript here: http://www.dovepress.com/therapeutics-and-clinical-risk-management-journal 